\title{
APPLICATION OF DIELECTRIC RESONATORS TO SURFACE IMPEDANCE MEASUREMENTS OF MICROWAVE SUSCEPTORS
}

\author{
M. Celuch ${ }^{1}$, J. Rudnicki ${ }^{1}$, J. Krupka ${ }^{2}$, W. Gwarek ${ }^{1}$ \\ ${ }^{1}$ QWED Sp. z o.o., Krzywickiego 12/1, 02-078 Warsaw, Poland \\ ${ }^{2}$ Faculty of Electronics and Information Technology, Warsaw University \\ of Technology, Nowowiejska 15/19, 00-665 Warsaw, Poland \\ mceluch@gwed.eu
}

Keywords: microwave susceptors, active packaging, material measurements, microwave dielectric resonators, microwave heating

\begin{abstract}
This paper describes the application of dielectric resonators (DR) to the measurements of surface impedance of microwave susceptors. We demonstrate that the single-post (SiPDR) configuration is applicable to plain susceptors before use, while the split-post (SPDR) configuration - to crazed susceptors after use. Attention is given to the full characterisation of active packaging, that is, the influence of paper support on the overall electric losses is also investigated. The measurements can be preformed with various form-factor VNAs, including benchtop VNAs and hand-held FieldFox, though the most economical setup is constructed with a dedicated computer-controlled microwave signal oscillator system available under the name of Q-Meter. Finally, an extension of dielectric resonator measurements to surface imaging is presented, achieved by incorporating the resonator in a $2 \mathrm{D}$ automatic scanner.
\end{abstract}

\section{Susceptors and their microwave characterisation}

Microwave susceptors are thin metallic layers incorporated into microwaveable food packages for the enhancement and control of the heating process [1], [2], [3]. Their most typical function is to increase the microwave power dissipation at the specific stages of the heating, though in some cases they are used to shield selected parts of the food. A daily-life example is a microwave popcorn bag: at the room temperature, the seeds of corn and the solidified grease are both lossless, and it is only thanks to the susceptor that the microwave heating initiates. The grease (and then the corn) warm up by the classical heat diffusion, their electric losses increase, and volumetric heating begins. The susceptor, on the other hands, breaks due to thermal expansion of its supporting material, no longer conducts the surface currents, and hence no longer heats [4].

The parameter which fully characterises a microwave susceptor, from the viewpoint of its microwave behaviour, is surface resistance - also called sheet resistance - denoted by symbol $R_{s}$ and expressed in Ohm per square. For a plain uniform susceptor of thickness $d_{0}$ and conductivity $\sigma_{0}$ we have:

$$
R_{s}=\left(d_{0} \sigma_{0}\right)^{-1}
$$

In a separate study to be presented at the conference, and based on [5], we mathematically prove that the actual values of conductivity and thickness are indeed irrelevant, and the effective value of $R_{s}$ unambiguously determines the power dissipated in the susceptor as well as its complex reflection and transmission coefficients, as long thickness $d$ of the surrogate 
layer modelling the original susceptor does not exceed ca. 0.02 wavelength $(1 \mathrm{~mm}$ at the ISM frequency of $2.45 \mathrm{GHz}$ ) and the following relation is conserved:

$$
R_{s}=\left(d_{0} \sigma_{0}\right)^{-1}=(d \sigma)^{-1},
$$

where $\sigma$ is the scaled conductivity of the surrogate.

Microwave measurements of surface resistance of susceptors are of practical importance for the understanding and design of microwaveable food packages. They have been subject of different approaches summarised, for example, in [1]. Traditionally those measurements are performed by transmission line methods: the susceptor is mounted between the flanges of two waveguide to coax adapters and its surface impedance is deembedded from Sparameters measured with a vector network analyser (VNA). Such setups require professional microwave laboratory equipment, which in turn needs to be operated by a trained microwave engineer. These deficiencies have stimulated our search for simpler and cheaper methods of susceptor measurements.

In this work, we discuss an approach based on dielectric resonator (DR) methods, originally developed for microwave measurements of low-loss dielectric materials. In particular, the split-post dielectric resonator (SPDR; Fig. 1) method after [6] has become a formal IEC standard [7] for low-loss laminar dielectrics. In [8], the SPDR method has been extended to high-resistivity semiconductor wafers and an alternative single-post dielectric resonator (SiPDR; Fig. 2) has been developed for semiconductors having resistivity less than $100 \Omega \mathrm{cm}$. While DR - just like waveguide - test fixtures can be operated with a VNA, they do not require all VNA capabilities. Hence to further decrease the cost and increase the easeof-use, we propose the DR setups operating with dedicated Microwave Frequency Q-Meters, controlled by a laptop [9].

The possibility to apply DRs to the characterisation of microwave susceptors has been noted in a workshop presentation [10] but remains unpublished and further unexplored. We build upon the concepts of [10] and discuss the application of SiPDR to plain susceptors before use, and of SPDR to crazed susceptors after use. We also present a new 2D scanner, which allows DR imagining of large inhomogeneous surfaces.

\section{Principles of SPDR and SiPDR application to susceptors}

The principle behind dielectric resonator characterisation of materials resides in making two measurements of resonant frequencies and Q-factors:

- one of the empty resonator, producing $f_{0}$ and $Q_{0}$,

- and one of the resonator loaded with the sample under test (SUT), producing $f_{m}$ and $Q_{m}$.

Dielectric resonators considered in this work are designed so that the electric field distribution inside the empty resonator denoted by $E_{0}$ remains approximately unchanged, after SUT is inserted, and constant along the SUT's height $h$. The following relations then apply, which allow calculating the complex permittivity $\varepsilon_{m}=\varepsilon_{m}{ }^{\prime}-\mathrm{j} \varepsilon_{m}$ " of the SUT material:

$$
\begin{aligned}
& \frac{f_{0}-f_{m}}{f_{0}} \approx \frac{h}{2 C} \iiint_{S}\left[\varepsilon_{m}^{\prime}(x, y)-1\right]\left|E_{0}(x, y)\right|^{2} d S \\
& \frac{1}{Q_{m}}-\frac{1}{Q_{0}} \approx \frac{h}{C} \iint_{S} \varepsilon_{m}^{\prime \prime}(x, y)\left|E_{0}(x, y)\right|^{2} d S
\end{aligned}
$$




$$
C=\iiint_{V}\left|E_{0}(x, y)\right|^{2} d V
$$

where $V$ is DR's volume and $S$ is SUT's lateral surface. The above equations can be manipulated to provide the value of surface resistance of thin material sheets deposited on semi-insulating substrates, in which case the resonator loaded with the semi-insulating substrate is considered as the "empty" one. While the nonlinear manipulations exceed beyond the scope of this paper, their graphical plots can be found in e.g. [8].

In the split-post dielectric resonator configuration after [6], the electric field demonstrates maximum strength in the area between the two dielectric posts. Hence, there is a strong interaction between the resonator and the SUT. The SPDR method has therefore been recognised as a standard for the characterisation of low-loss dielectric materials [norm], on average providing the accuracy of $0.3 \%$ for real permittivity and $3 \%$ for the loss tangent (see [7][11] for more detailed error estimates). For higher-loss materials, the resonance is damped and thereby the highest measurable conductivity is of the order of $1 \mathrm{~S} / \mathrm{m}$, corresponding to the lowest sheet resistance of $5 \mathrm{k} \Omega /$ sq. Hence SPDRs can be used for typical susceptors after use or during use. For plain susceptors before use, whose sheet resistance is expected to be significantly below $5 \mathrm{k} \Omega / \mathrm{sq}$, an alternative single-post configuration (SiPDR) previously developed for semiconductor wafers [8] becomes applicable. In SiPDR the electric field decays rapidly in the direction from the dielectric post towards the metal plate, where it sets to zero, see Fig. 2. The sample weakly interacts with the resonant fields allowing one to measure sheet resistances as low as $0.2 \mathrm{~m} / \mathrm{sq}$.
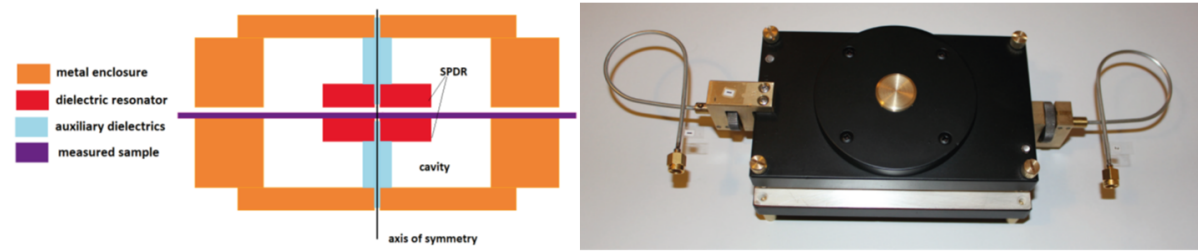

Fig. 1. Split-Post Dielectric Resonator: schematic (left) and photo (right) of an example implementation [11].
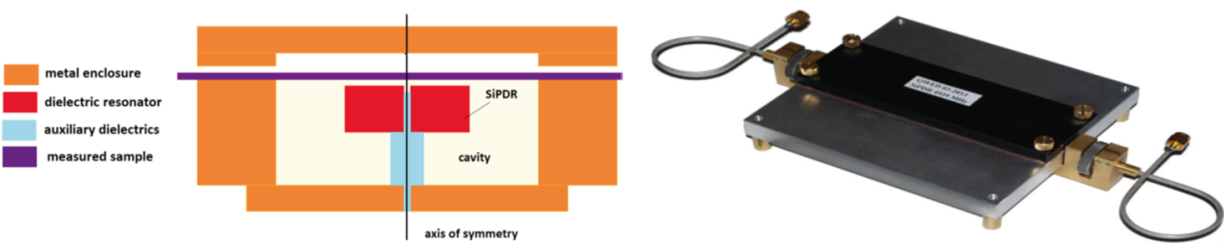

Fig. 2. Single-Post Dielectric Resonator: schematic (left) and photo (right) of an example implementation [11].

\section{Example measurements of microwave popcorn packages}

To validate our existing SPDR and SiPDR in application to microwave susceptors, we bought a few tens of ready-to-use packages of popcorn. We divided them into several batches of four bags each. For each batch, the smallest and biggest values of the measured surface resistance are recorded in Table 1. 
From the packages of the first batch, we cut out the part of the paper bag containing the susceptor and measured it in SiPDR (measurement in SPDR was impossible, due to too high losses of the sample). The following three batches were heated for, respectively, $60 \mathrm{sec}$, $120 \mathrm{sec}$, and $240 \mathrm{sec}$; then the part of the bag containing the susceptor was cut out and measured in SPDR. (Here, some of the samples could be measured in SiPDR, but at the limit of SiPDR application, where high sensitivity to measurement conditions led to big deviations between the results.)

All the above four batches of the measurements characterised "susceptor on paper" and are recorded in the second column of Table 1. The values for the original unheated bags are in agreement with our expectations based on [1][10]. However, during the heating the surface resistance increases slower than expected in [10]. This could be explained by the contributions of losses from the paper, which is in contact with grease and becomes wet and sticky after the heating. To verify this hypothesis, we treated further batches analogously as above, but after the heating, we decomposed each package and measured the actual PET susceptor and the paper substrate separately. The results are recorded in the fourth and third columns of Table 1, respectively. They confirm that after $240 \mathrm{sec}$ of the heating, the greased paper and the crazed susceptor equally contribute to the overall electric losses of the package, which (based on the equations from [5]) cause by over an order in magnitude less of dissipated power than the original unheated susceptor.
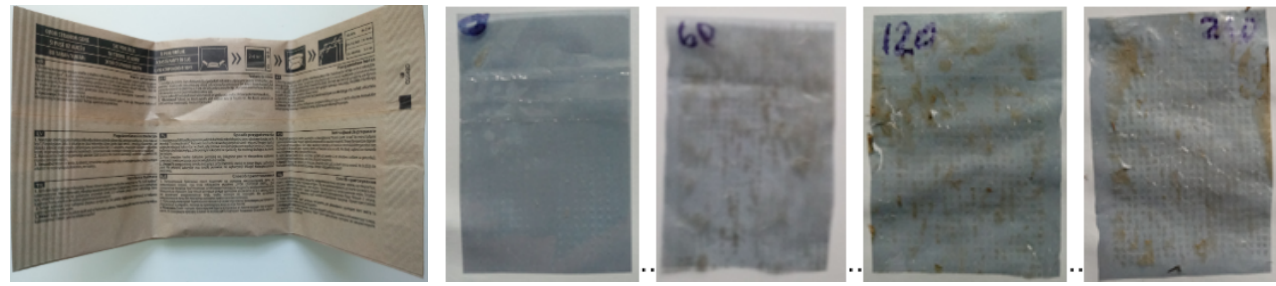

Fig. 3. From left to right, examples of: popcorn bag, original unheated susceptor and susceptors heated for $60 \mathrm{sec}, 102 \mathrm{sec}, 240 \mathrm{sec}$.

Table 1. Measurements (blue - SPDR, red - SiPDR)) of surface resistivity [ $\Omega / \mathrm{sq}]$ of the extracted elements of popcorn packages: susceptor on support paper; only paper, and only susceptor. Results in blue are from SPDR, red from SiPDR. From each batch of the measurements, minimum and maximum values are recorded.

\begin{tabular}{cccc}
\hline $\begin{array}{c}\text { Heating time } \\
{[\mathrm{sec}]}\end{array}$ & Susceptor on paper & Paper & Susceptor \\
\hline 0 & 141.10 & 86432 & 194.13 \\
0 & 258.75 & 87197 & 254.97 \\
60 & 3398.0 & 90233 & 2245.0 \\
60 & 4682.9 & 92795 & 3054.9 \\
120 & 7231.2 & 92265 & 4721.3 \\
120 & 8085.2 & 92993 & 5510.0 \\
240 & 6691.4 & 92559 & 9868.4 \\
240 & 6875.3 & 94605 & 11893.0 \\
\hline
\end{tabular}




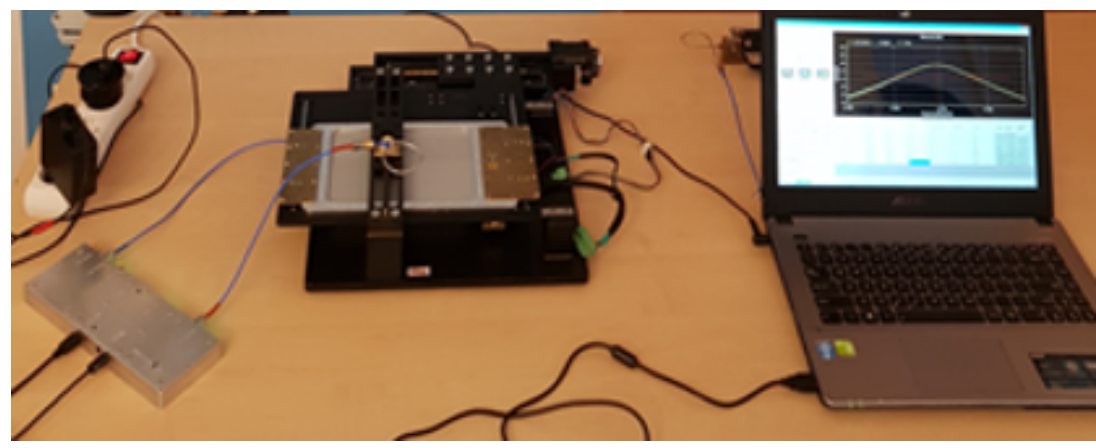

Fig. 4. A complete 2D surface imaging setup comprising Q-Meter, scanner, and laptop.

\section{IV.Conclusions and future work}

In the present work our existing split-post and single-post dielectric resonators, originally developed for low-loss laminar dielectrics and for resistivity measurements of semiconductor wafers at a nominal frequency of $5 \mathrm{GHz}$, have been applied to the measurements of surface resistance of microwave susceptors. It has been shown that the susceptors found in ready-to-use microwaveable popcorn packages are within the measurability range of SiPDR. Their surface resistance obtained with SiPDR and a dedicated Q-Meter is of the order of $100 \div 200 \Omega / \mathrm{sq}$, which is consistent with the more classical transmission line measurements requiring a professional VNA [1]. The susceptors crazed after use have been measured in SPDR, with surface resistances as high as $10 \mathrm{k} \Omega / \mathrm{sq}$ - which demonstrates a broader range of measurable values, when compared to the reported transmission line measurements performed up to $400 \Omega / \mathrm{sq}[1]$.

Our measurements taken with the Q-Meter have been confirmed in a laboratory setup consisting of the same resonators and a fully-fledged benchtop VNA. Both measurement setups produce the results in excellent agreement. Therefore, a suite compring one Q-Meter and two replaceable resonators (SPDR and SiPDR) is recommended for fast and easy characterisation of microwave susceptors, also in a manufacturing or environment food research laboratories where VNAs are unavailable. The proposed suite is low-cost (especially when compared to a VNA purchase), portable, and controlled from a standard laptop.

Finally, in Table 1 we note ca. 20\% deviations between the measurements of different susceptors, heated for the same time. Since each susceptor was measured only once, we cannot say whether those were the differences between the different susceptors, or any individual susceptor may exhibit such variations over its surface. Therefore, further work is planned on investigating surface non-uniformities of susceptors. To this end, surface imaging of susceptors will be performed with a newly constructed 2D scanner, which incorporates a $10 \mathrm{GHz}$ SPDR, as shown in Fig. 4. Microwave measurements can be taken with a Q-Meter or a new hand-held FieldFox VNA from Keysight; in both cases, the setup remains portable and controlled from a laptop. Extended results of the work will be presented at the Conference. 


\section{Acknowledgement}

This project has received funding from the European Union's Horizon 2020 research and innovation programme (H2020-NMBP-07-2017) under grant agreement MMAMA No. 761036.

\section{References}

1. M. R. Perry and R. R. Lentz, "Susceptors in microwave packaging," in Development of packaging and products for use in microwave ovens, $1^{\text {st }}$ ed., W. Lorence and P. S. Pesheck, London, United Kingdom: Woodhead Publishing Limited and CRC Press, 2009, pp. 207-236.

2. T. H. Bohrer, "Shielding and field modification - thick metal films," in Development of packaging and products for use in microwave ovens, $1^{\text {st }}$ ed., W. Lorence and P. S. Pesheck, London, United Kingdom: Woodhead Publishing Limited and CRC Press, 2009, pp. 237-266.

3. P. Risman, "Modelling the effects of active packaging of microwave foods," in Development of packaging and products for use in microwave ovens, $1^{\text {st }}$ ed., W. Lorence and P. S. Pesheck, London, United Kingdom: Woodhead Publishing Limited and CRC Press, 2009, pp. 349-371.

4. P. Risman, "Microwave popcorn popping - a multi-step process", private communication, 2019.

5. M. Celuch, K. Wilczynski, and M. Olszewska-Placha, "Macroscopic models of thin conductive layers: systematic evaluation for microwave heating and shielding applications", Proc. IEEE Intl. Microwave Symp. IMS 2019, Boston, MA, June 2019, pp. 47-50.

6. J. Krupka, A. P. Gregory, O. C. Rochard, R. N. Clarke, B. Riddle, and J. Baker-Jarvis, "Uncertainty of complex permittivity measurements by split-post dielectric resonator technique", J. Eur. Ceramic Soc., vol. 21, 2001, pp. 2673-2676.

7. IEC61189-2-721: 2015 standard.

8. J. Krupka and J. Mazierska, "Contactless measurements of resistivity of semiconductor wafers employing single-post and split-post dielectric-resonator techniques," IEEE Trans. Instr. Meas., vol. 56, no. 5, Oct. 2007, pp. 1839-1844.

9. P. Korpas, L. Usydus, and J. Krupka J (2012), "Automatic split post dielectric setup for measurements of substrates and thin conducting and ferroelectric films", Ferroelectrics, 434:1, pp. 113-120, 201. DOI: 10.1080/00150193.2012.732779

10. W. Gwarek, "Modeling and measurements of susceptors for microwave heating applications", at: IEEE IMS Workshop "Recent Advances in Microwave Power Applications and Techniques RAMPAnT', Boston, MA, June 2009.

11. http://www.qwed.eu/resonators.html\#ResonatorsFamily, accessed 10 June 2019. 